\begin{tabular}{|c|c|c|c|c|}
\hline Wash. & $\mathbf{M}$ & du. & & $\alpha$ \\
\hline March & $i$ & $10^{h}$ & 4 & $\pi_{2} 29^{s} 53$ \\
\hline & 8 & & 3 & 47,65 \\
\hline & 9 & & 3 & 6,53 \\
\hline & 10 & & 2 & 26,20 \\
\hline & 11 & & 1 & 46,70 \\
\hline & 12 & & 1 & $8,0 j$ \\
\hline & 13 & 10 & 0 & 30,30 \\
\hline & 14 & 9 & 59 & 53,47 \\
\hline & 15 & & 59 & 17,60 \\
\hline & 16 & & 58 & 42,72 \\
\hline & 17 & & 58 & 8,86 \\
\hline & 18 & & 57 & 36,03 \\
\hline
\end{tabular}

$\begin{array}{rr}+12 " \\ +13 & 16 “ 6 \\ 18 & 17,7 \\ 22 & 40,8 \\ 26 & 58,4 \\ 31 & 10,4 \\ 35 & 16,7 \\ 39 & 17,1 \\ 43 & 11,8 \\ 47 & 0,3 \\ 50 & 42,6 \\ 54 & 18,6\end{array}$

\begin{tabular}{r} 
logy \\
\hline 0,35195 \\
35298 \\
35407 \\
35520 \\
35639 \\
35762 \\
35890 \\
36022 \\
36158 \\
36299 \\
36445 \\
36594
\end{tabular}

\begin{tabular}{c|}
$\frac{\log r}{0,50597}$ \\
50620 \\
50643 \\
50666 \\
50689 \\
50711
\end{tabular}

Wash.M.Midn.

$\underbrace{\text { Wash.M.Midn. }}_{\text {March } 19} \underbrace{\alpha}_{9^{15} 57^{m}}$

$20 \quad 56 \quad 33,53$

$21 \quad 56 \quad 3,90$

$22 \quad 5535,40$

$23 \quad 55 \quad 8,04$

24. 5441,84

$25054 \quad 16,84$

$26 \quad 53 \quad 53,08$

$\begin{array}{llll}27 & 9 & 53 & 40,58\end{array}$

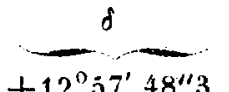

$+$

13111,5

428,1

$\begin{array}{lll}7 & 37,9\end{array}$

1041,0

1337,2

1626,4

198,

$+132143,6$

$\log \Delta$

T2,36747

36094

37065

37229

37397

37569

37743

37921

8 Felir. $204^{\text {h }} 5^{\text {"n }} 8$ Wash. M. T.

Inteusity of light $=0,64$.

Verzeicimss der Instrumente, welche in dem optischen Institute von G. of S. Merz, vormals Utzschneider $\$$ Fraunhofer in Hinchen,

für nachstehende Preise geliefert werden.

(Schluss ron ri 1345. )

Tuben, traybare, mit messingener säule auf 3 Füssen, init ficiner Verticalbewegung:

Nr. 22 Tubus von 43 Lim. Ocfin, 54 Zoll Brennw, mit 2 Guld. irdisclren Ocalaren ron $60-$ und $90-, 5$ astronomischen von 48-, 72-, 108-, $162-$ n. 243mal. Vergr., 2 Sonnenglïsern und Sucher ...........

23 Tubus von 37 kin. Oeffn., 48 Zoll Brennw, mit 2 irdischen Ocularen ron $57-$ und 80 -, und 4 nstronomischen von 64-, 96., 144- und 216 mal Vergs. und Sonnenglas................

24 Tubus von 34 Lin. Oeffn, 42 Zoll Bremw., 2 irdisthen Ocularen ron 50 - und 70 , und 3 astronomisthen von 54-, 84- und 120 maliger Vergr. und Sonnenglas. . . . . . . . . . . . . .

25 Tulus von 29 Lin. Oeffin, 30 Zoll Brennw., mit 1 irdischem Oculare von 42-, und 2 astronomischen von 60 . und $90 \mathrm{mal}$. Vergr. und Sonnenglas. Das Fernohr hat nessingene Röhre...........

26 Tubus greich dem vorhergehenden mit Ausnahme einer feinen vertiealbewegung . . . . . . . .

27 Tulbus von 21 Lin. Oeffn., 20 Zoll Bremu, mil 1 irdischen Oculare ron $32-$. und 2 astronomischen von 40- und $60 \mathrm{mal}$. Vergr. Im kebrigen gleich

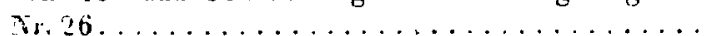

Tuben ohne Stativ uit hohren rou Mabagony, in Kästen von Fichteuholn:

28 Tubus von 34 Lin. Oeffn., 48 Zoll Brennw., mit 1 irdischen Oculare ron $62-$, und 3 astronomischen von 64-, 96- und $144 \mathrm{mal}$. Vergr. and Sonnenglas.

29 Tubus von 32,5 Lin. Oeffn., 42 Zoll Brennw., mit 1 irdisclien Ocnlare von 55 , und 2 istronomiseben von 84- und 126 mal. Vergr. nebst Sommenglas...

30 Tubus von 27 Lin. Oeffn., 42 Zoll Breunw., wit 1 irdischem Oculare von 42-, und 2 astronomischen von 60. und $90 \mathrm{mal}$. Vergr. und Sonnenglas ....
See- und Marine-Fernmöhren nit Röhren von Mahagony, die zwei ersten in fichtenen Küsten, die andern in Papp-Etuis:

Nr. 31 Sceferuroler von 29,5 Lin. Oefin., 42 Loll Bremnw., Guda.

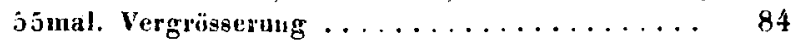

32 Seeferurohr von 25,5 Lin, Oeffir, 30 Zoll Brennw., $42 \mathrm{mal}$. Vergrösserung. . . . . . . . . .

33 Secfornohr von 21 Lin. Oefin., 24 gonl Brenuw., 39 mal. Vergr. . . . . . . . . . . . . .

34 Seeferurohr von 19 Lin. Oeffn., 20 Zoll Brenmw. 32 mal. Vergrössermg. . . . . . . . . . .

35 Secferurohr vou 16 Lin. Oeffn., 16 Zoll Brenuw., 26 mal. Vergrössemung . . . . . . . . . .

36 Seefermpohr von 10 Lin. Oeffn., 10 Zoll Brennw.. $14 \mathrm{mal}$. Vergrüsserung. . . . . . . . . . .

37 Marinefermoler von $16 \mathrm{Li}$, Oeffn., 16 \%oll Bremw.. 12 mal. Ver rrösserung. . . . . . . . . . .

38 Marineferurohr, wie das vorhergehende, aber mit verstellbarem Ocnlare, von 12-20mal. Vergr...

'Zugfermä̈hren mit häkerner Röhre und messingenen Auszügen, die ganze länge nn cinige Zoll grösser als die Brennweite. sanmmtlich in Ftuis von Maroquin :

39 Zugferurohr von 29 Lin. Oeffn., 30 Znll Brennw., $72 \mathrm{mal}$. Verge, und 5 Auszageröhren........

40 Zugferurohr von 24 Lin, Oefrn,, 24 Zoll Bremw., 39 mal, Vergr. und 4 Auszugsröhren.........

41 Zagferurohr von 21 Lin. Ocfin, , 20 Zoll Brennw., 32 mal. Vergrr. und $f$ Auszugsröhren.........

42 Zugfernrohr von 19 Lin Oeffu., 20 Zoll Brennw., $28 \mathrm{mal}$. Vergr. und 3 Auszugsröhren.........

43 Zugfernrohr von 16 Lin. Oeffin., 16 Zoll Brennw., $26 \mathrm{mal}$. Vergr. lind 3 Auszugsröhren........
56 $\log r$

0,50733

50755

50777

50799

0,50821 
Nr. 44 Ingferurohr won 13 Lin. Oefin., 13 Zoll Brennw., Guld. $18 \mathrm{mal}$. Verge. und 3 Auszugsröluren........ 21

45 Zugferurohr von 10 Linien Oeffn., 6 Zoll Brennw., 14 mal. Vergr. und 3 Auszugsröhren........ Orthuscopische Oculare erhöhen die Preise je um iff. und werden nur auf besundere Bestellung dazu geliefert.

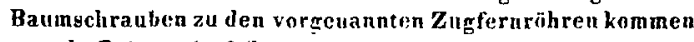
je nach Grösse $4-6 \mathrm{fl}$.

Fernmolne-Objective, astronomische :

46 Olijectiv von 10 Linien freier Ocffnung....... $47=11=\quad=\quad=\ldots \ldots \ldots$ $48=: 12=\quad=\quad=\ldots \ldots \ldots$ $49=\quad: 13$

$50=14$

51

52

53

Bei grösseren Dimensioneu als $10 \mathrm{Zoll}\left(120^{\prime \prime \prime}\right)$ wird nan sich über die Preise vereinigen.

\section{Microseope, ausammengesetze.}

Der Tubus allein hat die nöthige grobe und feinc Bewegung. Der Belenchtungespiegel ist auch mor schiefen Beleuchtung eingerichtet. Beigegeben sind cine gehörige Anzahl Ohjesten- und Deck-Gläser :

71 Microscop wit 8 Ohjertiv-Systemen und 5 Ocularen 20- bis 1800 mal. Vergr, einem Schrabenmiciometer, welches noch 0,0001 eines Pariser Znlles messen lässt, drchbarem Objectentische, Zeichnnngsprisma, Compresurium, 2 Nicol'schen Prizmen znr Polariaation etc. .
Nr.72 Nicroscop mit 6 Objectiv-Systemen und 5 Ocularen, Guld. von 20 - bis $1200 \mathrm{mal}$. Vergr., drehbarem Objectentische, Ocular- und Objectiv-Micrometer, Zcichnungsprisma, Compressurium und Polarisationsapparat..

73 Nicroscop mit 4 Objectiv-Systemen und 3 Ocularen von 40 - bis 900 mal. Vergr., drehbarem Objectentische und Ocularmicrometer ............

74 Microscop nit 2 Ohjectiv-Systemen und 3 Ocularen von 60 - bis $600 \mathrm{mal}$. Vergrösserung.........

75 Microscop, wie das vorhergehende, in einfacberer Aufstellung . . . . . . . . . . . . . . .

76 Handmicroscop mit 50 - und $100 \mathrm{mal}$. Vergr......

77 Objectiv-Systeme (gewöhnliche und Systemes d'immersion) von $1 ", \frac{1}{2}{ }^{\prime \prime}, \frac{1}{3} ", \frac{1}{4}{ }^{\prime \prime}, f^{\prime \prime}, \frac{1}{9} ", \frac{1}{1}{ }^{\prime \prime}, \frac{1}{15}$ Brennweite ......................... 14-70

78 Lupen von 5-, 12-, 17-, 24- und 32mal. Vergr.... $3 \frac{1}{2}$

\section{Verschiedene Gegenstände.}

79 Heliostat mit Uhrwerk, Stunden- und Declinations-

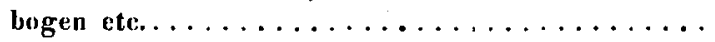

80 Apparat zur Beobachtung der physich-optischen Experimente in Bezug auf die Entdeckung Fraunhofer's über Brechung, Farbenyerstreuung, Beugung des Lichtes, Hervorbringung der Farbenspectra etc...

\section{Kreis-Nicrometer}

a) mit doppeltem Stahlringe $\ldots \ldots \ldots \ldots$

b) mit einfachem Stahlringe $\ldots \ldots \ldots \ldots$

82 Repetireude Filar-nicrometer mit und ohne Lampen und Positionskreis . . . . . . . . . . . .

83 Heliotrope zum Behuf geodätischer Messungen .... 84 Licht - Polarisirungs - Apparate . . . . . . . . .

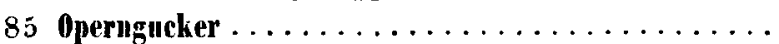
86 Photographische Linsen...............

87 Prismen vol Crowu- und Plintglas, einfache und zusammengesetzte . . . . . . . . . . . . .

88 Plan- und Parallel-Spiegel. ...........

89 Oculare, irdische und astronomische, anch blosse Ocularlinsen . . . . . . . . . . . . .

90 Libellen uir auf Bestellung verfertigt und leren Preise nach Maasgabe ihrer Dimensionen bestimmt.

\section{I h a l t.}

(Ziı N. 1840.) Zeichnungen der Sonnenflechen und Fackelu vor und nach der totalen Sonnenfinsterniss des 18. Juli 1861. Von Herrn Dr. G. Schweizer, Director der Steruwarte in Moskau 49. Carl Friedrich Gauss' Werke. Herausgegeben von der Königl. Gesellschaft der Wissenschaften zu Göttingen 53 . Anzeige eines Druckfehlers in allen Cometenverzeichnissen 61.Schreiben des Herrn Webb, Mitglied des Council der Royal Astronom. Soc., an den Herausgeber 61. Beobachtungen der Calypso. 1) zu Bilk ron Herrn Dr. Kr. Luther. 2) zu Mannbeim von Herrn Prof. Schönfeld 63. -

(Zu Nr. 1349.) Schreiben des Herm Simon Newcomb an den Herausgeber 65. -

Work oil Asteroids by $E$. Schubert. Communicated by J. WTinlock Esq., Superintendent of American Ephemeris 67: -.. Preis-Courant des optischen Instituts der Herren $G$. \& $s$. Merz 13. - 